\title{
铝合金基底微碳球作为润滑油添加剂的摩擦学 性能及其润滑机理
}

\author{
古毓康 ${ }^{1}$ ，曹 䂞 ${ }^{1}$ ，万勇 ${ }^{1}$, 高建国 ${ }^{2}$ \\ (1. 青岛理工大学 机械工程学院, 青岛 266520; 2. 山东出入境检验检疫局 检验检疫技术中心, 青岛 266001)
}

摘 要: 采用绿色水热制备方法，以葡萄糖为前身化合物一步制得直径在 400 500 nm、尺寸均匀的单分散微碳球, 采用扫描电子显微镜、红外光谱等手段对微碳球的表面形貌及化学特性进行表征, 利用多功能摩擦磨损试验机 考察了微碳球作为润滑油添加剂在铝合金一钢摩擦副的减摩耐磨特性，探讨了微碳球的润滑作用机理。结果表明: 将微碳球作为液体石蜡和商用机油润滑添加剂可以显著提高铝合金-钢摩擦副上的摩擦学性能, 这主要是因为 在滑动过程中微碳球可能进入到接触区，阻止摩擦副之间的直接接触，并起到纳米滚珠作用。

关 键 词: 摩擦学性能; 微碳球; 润滑油添加剂; 铝合金

中图分类号: TH117 文献标识码: A

\section{Tribological Performance and Lubrication Mechanism of Carbon Microspheres as Oil-based Lubricant Additive on Aluminum Alloy Substrate}

\author{
GU Yu-Kang ${ }^{1}$, CAO Lei ${ }^{1}$, WAN Yong ${ }^{1}$, GAO Jian-Guo ${ }^{2}$
}

(1. School of Mechanical Engineering, Qingdao University of Technology, Qingdao 266520, China; 2. Inspection and Quarantine Center, Shandong Exit \& Entry Inspection and Quarantine Bureau, Qingdao 266001, China)

\begin{abstract}
A one-step green hydrothermal method was used to prepare carbon microspheres with size of 400-500 nm by using glucose as the precursor compound. Morphologies and chemical properties of the carbon microspheres were characterized by means of SEM and FTIR. Their tribological properties as the lubricant additive were evaluated on aluminum-steel sliding contact by using a multi-functional friction and wear tester. The lubrication mechanism was also discussed. The results indicate that the carbon microspheres possess good friction-reducing and antiwear performance when they are used as the lubricant additive in both liquid paraffin and 0W-40 engine oil on aluminum-on-steel pairs. Carbon microspheres may enter the contact region and prevent the direct contact of the friction pair. At the same time, they may play a role as micro-nanospheres between the sliding contacts to provide an excellent lubricating effect.
\end{abstract}

Key words: tribological properties; carbon microspheres; lubricant additive; aluminum alloy

铝及其合金比重小、质量轻，比强度和比刚度 较高, 并且具有良好的延展性、可塑性和耐蚀性，广 泛应用于日常生活以及航空航天、汽车、机械制造
等工业领域 ${ }^{[1-5]}$ 。然而, 由于铝合金硬度较低且化学 活泼性较高，因而铝合金的耐磨和抗擦伤能力差, 特别是在常用的铝-钢接触条件下，摩擦副之间存

收稿日期：2016-08-08; 收到修改稿日期：2016-09-23

基金项目：国家自然科学基金(51375249); 科技部质检行业公益项目(201410083)

National Natural Science Foundation of China (51375249); General Administration of Quality Supervision, Insection and Quarantine of the People's Republic of China (201410083)

作者简介：古毓康(1991-), 男, 硕士研究生. E-mail: yukang_gu@163.com

通讯作者: 万 勇, 教授. E-mail: wanyong@qut.edu.cn 
在较大的固溶度，易发生铝表面向钢对偶的转移， 使得铝件磨损严重 ${ }^{[6-10]}$ 。因此, 铝合金润滑的研究引 起了广泛关注。Liu 等 ${ }^{[11-13]}$ 发现 P-N 型极压抗磨添 加剂以及含 $\mathrm{N} 、 \mathrm{O}$ 元素的有机添加剂在铝-钢摩擦副 中具有良好的润滑性能, 可以有效提高铝合金的摩 擦学性能。Xia 等 ${ }^{[14]}$ 讨论了多种酰胺化合物作为液 体石蜡润滑添加剂在铝-钢摩擦副上的摩擦磨损性 能; Wan 等 ${ }^{[15-17]}$ 研究了含磷、含氯等不同的润滑添 加剂在铝-钢摩擦副上的润滑性能; Das 等 ${ }^{[18]}$ 研究了 铝硅合金与钢对磨过程中的边界润滑性能。除此之 外, Liu 等 ${ }^{[19-22]}$ 研究了多种离子液体在铝-钢摩擦副 中的润滑机理和摩擦学性能。

近年来, 迅速发展的碳纳米材料为开发新型润 滑材料提供了基础 ${ }^{[23-24]}$ 。碳纳米材料主要包括碳纳 米管、碳纳米纤维和纳米碳球, 其中碳球按尺寸大 小又可以分为直径在 2 20 nm 之间富勒烯族系、直 径 $50 \mathrm{~nm} \sim 1 \mu \mathrm{m}$ 之间的部分石墨化的纳米碳球和直 径 $1 \mu \mathrm{m}$ 以上的碳微珠 ${ }^{[25]}$ 。直径 $50 \mathrm{~nm} \sim 1 \mu \mathrm{m}$ 之间的 微碳球在表面形貌、硬度、耐热性和化学稳定性等 方面都具有优良的特性 ${ }^{[26-28]}$, 并且由于石墨是一类 天然固体润滑剂, 所以部分石墨化的微碳球本身具 备良好的固体润滑特性, 可以在摩擦表面形成易剪 切的表面润滑膜, 降低摩擦, 减小磨损。此外, 微碳 球呈现完美的球形结构, 且强度硬度较高, 可以在 接触区充当纳米滚珠, 在接触界面之间产生物理分 离, 隔离摩擦副, 并在一定程度上将滑动摩擦转化 为滚动摩擦, 起到润滑作用 ${ }^{[29-30]}$ 。另外, 微纳颗粒还 可能填补摩擦表面, 从而实现磨损区的自我修复。 因此, 微碳球有望作为一种新型的润滑添加剂得 到广泛应用。

本工作通过葡萄糖水热法一步制备微碳球, 研 究微碳球作为液体石蜡和商用全合成机油润滑添加 剂在铝合金一钢摩擦副上的减摩抗磨性能, 希望为 研制和开发具有良好润滑性能的铝合金专用润滑剂 提供一些借鉴。

\section{1 实验方法}

\section{1 实验材料}

采用 5052 铝合金(粗楉度为 $0.3 \mu \mathrm{m}$ ) 作为实验基
底材料，其组成成分如表 1 所示。

采用化学纯液体石蜡 (LP) 作为矿物基础油, 实 测 $21^{\circ} \mathrm{C}$ 下动力粘度为 $0.035 \mathrm{mPa} \cdot \mathrm{s}$ 。同时, 选用商用 $0 \mathrm{~W}-40$ 全合成机油作为微碳球润滑载体, 实测 $21^{\circ} \mathrm{C}$ 下动力粘度为 $133.2 \mathrm{mPa} \cdot \mathrm{S}$ 。

葡萄糖为上海埃彼化学试剂有限公司生产的分 析纯试剂。

\section{2 微碳球(CS)的制备}

参照文献[28]报道的方法采用水热法一步制 得微碳球。取 $1 \mathrm{~mol} / \mathrm{L}$ 葡萄糖溶液适量, 加入到聚 四氟乙烯内祄的钢制水热反应釜中， $180^{\circ} \mathrm{C}$ 保持 4 $5 \mathrm{~h}$, 随炉冷却至室温。对反应物进行反复清洗并离 心得到棕褐色固体, $80^{\circ} \mathrm{C}$ 干燥 $12 \mathrm{~h}$ 后充分研磨, 干 燥待用。

\section{3 润滑液的配制}

称取一定量的微碳球, 分别加入到液体石蜡 和商用机油中配置成含 $0.5 \mathrm{wt} \%$ 微碳球的混合润滑 液, 搅拌并超声震荡 $10 \mathrm{~min}$ 待用。为了改善微碳球 在基础油中的分散稳定性, 在液体石蜡中添加了 1vol\%的表面活性剂 Span-80, 在 $48 \mathrm{~h}$ 内未观察到 明显沉降。

\section{4 性能表征}

使用 UMT-3 多功能摩擦磨损试验机进行摩擦 实验, 上试样为 $\phi 9.5 \mathrm{~mm}$ 的 440-C 钢球, 下试样为 $\phi 69.5 \mathrm{~mm}$ 的铝合金基底。长磨实验的工况条件为: 施加载荷分别为 $10 、 30$ 和 $50 \mathrm{~N}$, 滑动半径 $24 \mathrm{~mm}$, 转速 $100 \mathrm{r} / \mathrm{min}$, 持续时间 $20 \mathrm{~min}$ 。实验中由试验机 记录实时摩擦系数变化情况。实验前使用石油醚、 酒精和去离子水分别对基底和钢球进行超声清洗 $10 \mathrm{~min}$, 氮气吹干。摩擦磨损实验后，分别用去离子 水将基底和钢球冲洗干净并用氮气吹干, 观察上下 试样表面磨痕形貌。

采用 Mitutoyo SJ-210 粗粘度仪对基底磨痕二维 形貌进行测量，通过积分计算得到磨损体积。采用 日本 Hitachi S-3500N 型扫描电子显微镜观察摩擦 结束后铝试样磨痕的表面形貌。使用实验室自制的 光干涉球盘点接触实验系统对微碳球润滑机理进行 探究。该装置采用单色光干涉法能够直观地观察到 微碳球在润滑接触区的实时状态。

表 15052 铝合金化学组成成分/wt\%

Table 1 Compositions of 5052 aluminum alloy/wt\%

\begin{tabular}{cccccccc}
\hline $\mathrm{Si}$ & $\mathrm{Fe}$ & $\mathrm{Cu}$ & $\mathrm{Mg}$ & $\mathrm{Cr}$ & $\mathrm{Mn}$ & $\mathrm{Al}$ \\
\hline 0.08 & 0.32 & 0.02 & 2.39 & 0.18 & 0.08 & Balance \\
\hline
\end{tabular}




\section{2 结果与讨论}

\section{1 微碳球的表征}

有研究表明, 水热条件下微碳球的形成要经过 葡萄糖分子的聚合反应、羟基脱水和最终碳化过程 等步骤才能形成微碳球颗粒 ${ }^{[30]}$ 。图 1 给出了微碳球 表面的 SEM 形貌, 由图可知, 微碳球表面光滑且呈 现完美的球形形状，颗粒尺寸在 400 500 nm 之间。

采用红外光谱技术对微碳球表面官能团进行表 征, 结果如图 2 所示。从图 2 可以看出, 波数在 3250 $3500 \mathrm{~cm}^{-1}$ 处有一较强吸收峰, 对应羟基或羧基中的 $\mathrm{O}-\mathrm{H}$ 的伸缩振动; 2750 3000 $\mathrm{cm}^{-1}$ 处有一小的吸收峰, 对应烷烃 $\mathrm{C}-\mathrm{H}$ 的伸缩振动; 波数 $1650 \sim 1800 \mathrm{~cm}^{-1}$ 处的 吸收峰, 对应羰基 $\mathrm{C}=\mathrm{O}$ 的伸缩振动; $1600 \sim 1700 \mathrm{~cm}^{-1}$ 处较小的吸收峰对应的是烯烃 $\mathrm{C}=\mathrm{C}$ 的伸缩振动; $1038 \mathrm{~cm}^{-1}$ 处的吸收峰, 对应芳香族 $\mathrm{C}-\mathrm{O}-\mathrm{C}$ 反对称 伸缩振动和对称伸缩振动。结果表明, 微碳球表面 存在由 $-\mathrm{OH} 、 \mathrm{C}=\mathrm{O} 、-\mathrm{COOH}$ 等组成的含氧官能团。

\section{2 摩擦学性能}

图 3 分别给出在载荷为 $10 \mathrm{~N}$, 液体石蜡及添加 微碳球的液体石蜡润滑时, 铝合金一钢摩擦副的摩

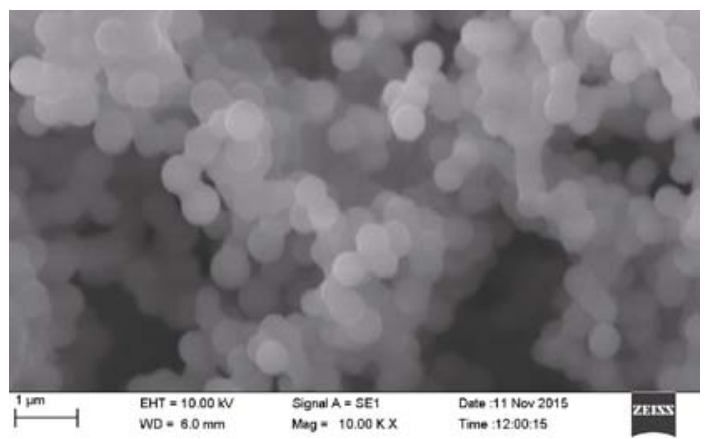

图 1 微碳球的 SEM 照片

Fig. 1 SEM image of carbon microspheres

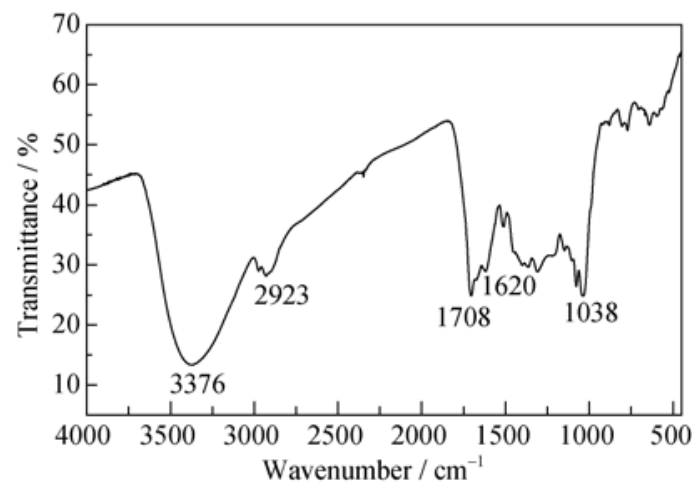

图 2 微碳球表面的 FT-IR 图谱

Fig. 2 FT-IR spectrum of carbon microspheres

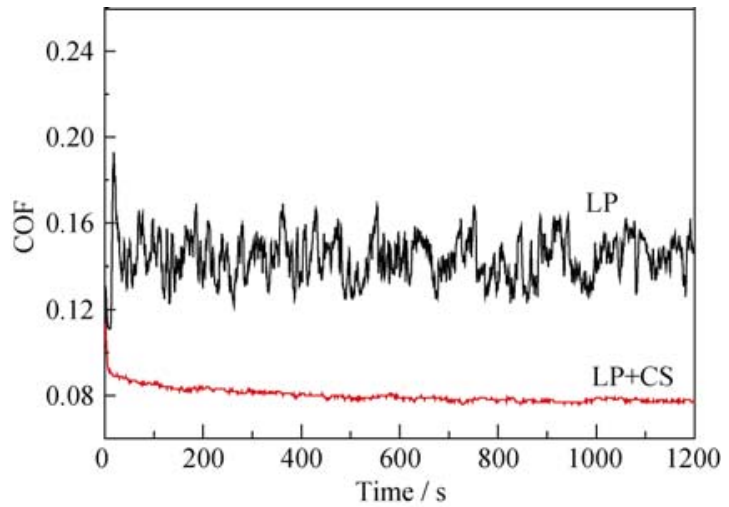

图 3 摩擦系数随滑动时间的变化曲线

Fig. 3 Evolution of frictional coefficient with sliding time

擦系数(COF)随滑动时间的变化曲线, 从图中可以 看出, 在液体石蜡中添加微碳球可以显著降低铝钢摩擦副的 $\mathrm{COF}$ 。在基础油润滑下, 铝-钢摩擦副的 $\mathrm{COF}$ 在 $0.13 \sim 0.16$ 之间反复波动, 摩擦行为不稳定, 平 均 $\mathrm{COF}$ 维持在 0.14 左右, 这说明液体石蜡并不能很 好的润滑铝-钢摩擦副。而在液体石蜡中添加微碳球 颗粒后, COF 降低至 0.08 左右，同时波动明显减小。

图 4 给出摩擦实验结束后通过二维轮廓测量得 到的铝试样磨痕二维轮廓图。通过磨痕深度及宽度 的对比可以明显看出，纯基础油润滑条件下，基底 磨损量很大，磨痕深度达到 $17 \mu \mathrm{m}$; 在基础油中添 加微碳球后, 材料的磨损量显著降低, 磨痕深度约 为 $2 \mu \mathrm{m}$ 。通过对磨痕横截面进行积分计算, 得到基 础油润滑时, 基底磨损量约为 $1.15 \mathrm{~mm}^{3}$; 添加微碳 球的液体石蜡润滑时, 基底磨损量仅为 $0.03 \mathrm{~mm}^{3}$, 磨损量仅为液体石蜡润滑时的 $2.6 \%$ 。

图 5 给出载荷增加至 $30 \mathrm{~N}$ 和 $50 \mathrm{~N}$ 时, 液体石 蜡及添加微碳球液体石蜡润滑时, 铝合金一钢摩擦 副的摩擦系数及磨损体积。在添加微碳球的液体石 蜡润滑时, 随着载荷的增加, 铝合金一钢摩擦副的摩

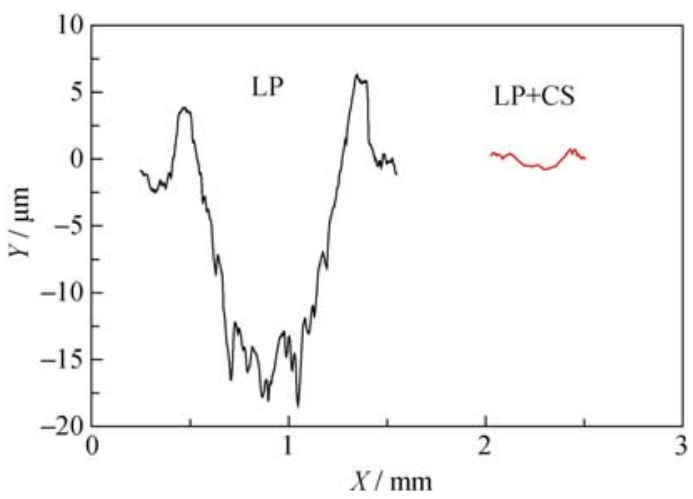

图 4 下试样磨痕二维轮廓图

Fig. 4 Surface profiles across the wear scans 


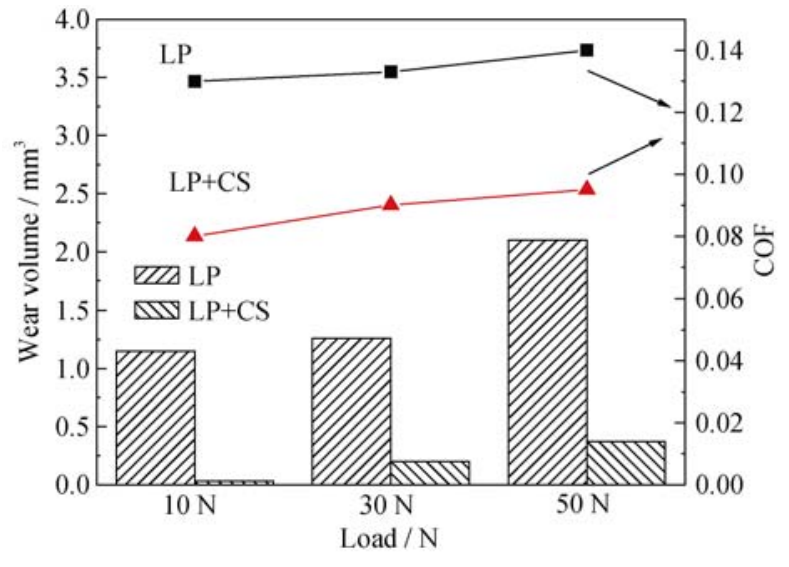

图 5 摩擦系数与磨损量随施加载荷的变化情况

Fig. 5 Change of friction coefficient and wear quantity with the applied load

擦系数略有增加, 但均保持在 0.10 以下，同时磨损 量也略有增大。即使在载荷 $50 \mathrm{~N}$ 条件下, 添加微碳 球的液体石蜡润滑下的磨损量也仅为液体石蜡润滑 下磨损量的 $14 \%$, 表明微碳球在高载下仍具有良好 的减摩和耐磨特性。

同时考察了微碳球在商用 $0 \mathrm{~W}-40$ 全合成机油 中的摩擦学性能, 结果示于图 6 和图 7 中。从图 6 中不难看出, 纯机油润滑时, COF 保持在 0.06 0.07 之间, 存在明显波动; 而在机油中添加微碳球后, $\mathrm{COF}$ 虽然没有明显降低，但其波动现象明显减轻。 图 7 给出摩擦实验结束后，铝合金基底磨痕断面的 二维形貌图, 经计算可知, 机油润滑时铝基底磨损 量约为 $0.075 \mathrm{~mm}^{3}$, 而添加微碳球的机油润滑时, 基底磨损量仅为 $0.018 \mathrm{~mm}^{3}$, 是机油润滑时磨损量 的 $24 \%$ 。

综合以上分析不难看出, 微碳球作为润滑油添 加剂可以明显改善铝合金-钢摩擦副的摩擦学性能。

\section{3 磨痕的 SEM 表征}

图 8 为摩擦实验结束后铝合金下试样磨痕的 SEM 形貌。图 8(a)和 8(b) 为纯液体石蜡润滑时试样 磨痕的 SEM 图像, 可以看出, 磨痕表面损伤比较严 重, 发生少量的剥落以及黍着、疲劳损伤, 存在沿滑 动方向的犁沟现象, 特别在磨痕边缘位置存在大量的 塑性变形、脆性断裂和脱落, 磨痕宽度达到 $1 \mathrm{~mm}$ 左 右。图 8(c)和 8(d)为添加微碳球的液体石蜡润滑时 试样磨痕的 SEM 图像, 磨痕表面相对光滑, 未发现 犁沟现象, 磨痕宽度仅为 $0.29 \mathrm{~mm}$, 这进一步说明 添加微碳球可以改善基础油的抗磨性能。在机油润 滑的情况下也可以得到同样的结论。图 8(e)和 8(f) 为机油润滑时试样磨痕的 SEM 图像, 磨痕表面存

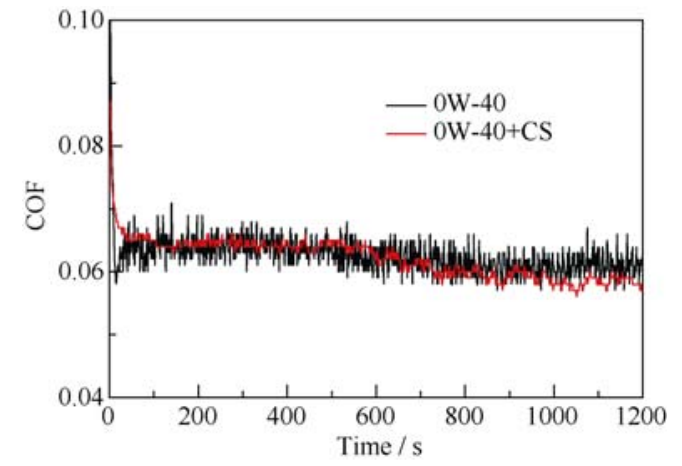

图 6 摩擦系数随滑动时间的变化曲线

Fig. 6 Frictional performance of different lubricants

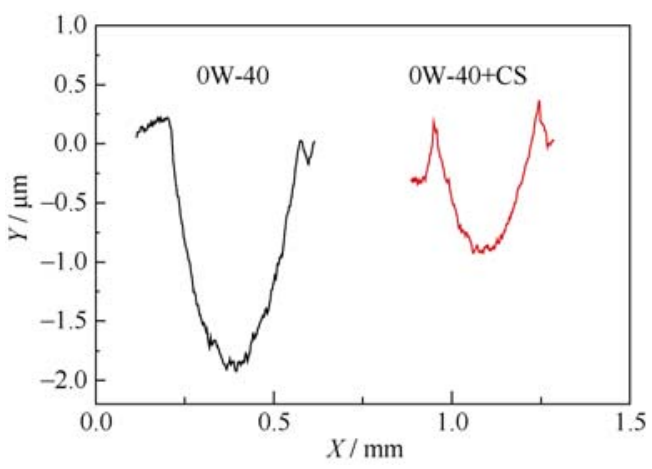

图 7 下试样磨痕二维轮廓图

Fig. 7 Surface profiles across the wear scans
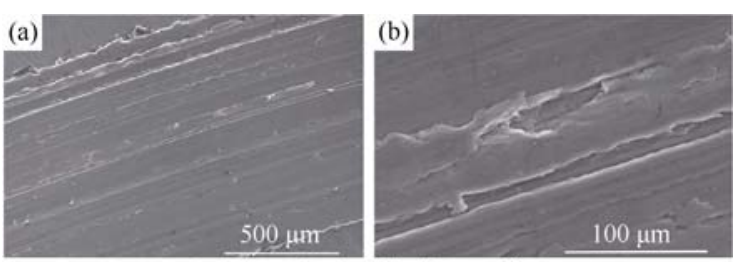

Lubricated using liquid paraffin
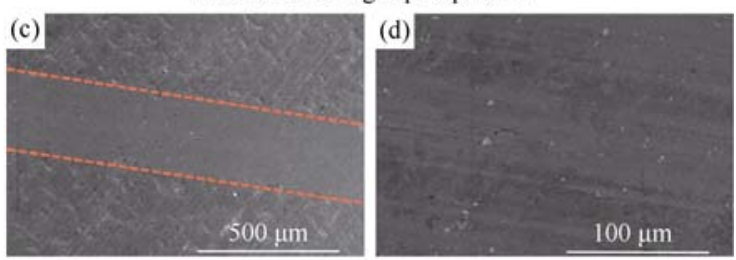

Lubricated using liquid paraffin with carbon microspheres
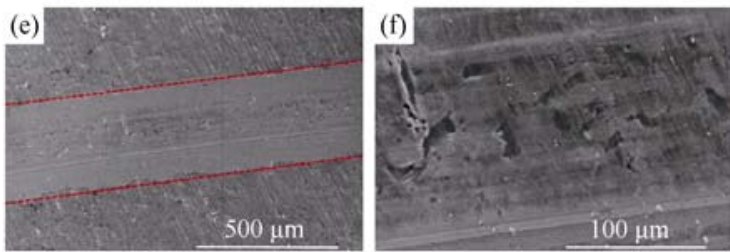

Lubricated using $0 \mathrm{~W}-40$ engine oil

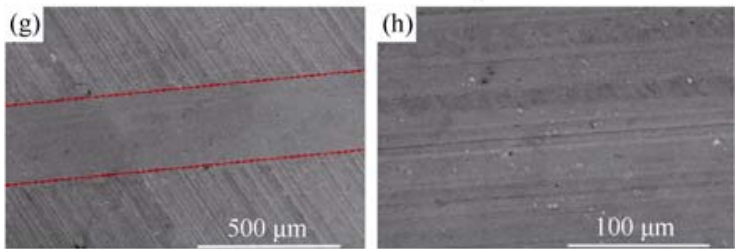

Lubricated using $0 \mathrm{~W}-40$ engine oil with carbon microspheres

图 8 磨痕表面的 SEM 照片

Fig. 8 SEM images of the worn surface 


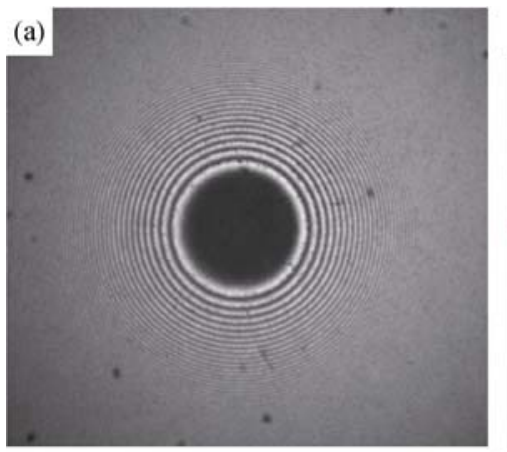

$0 \mathrm{~mm} / \mathrm{s}$

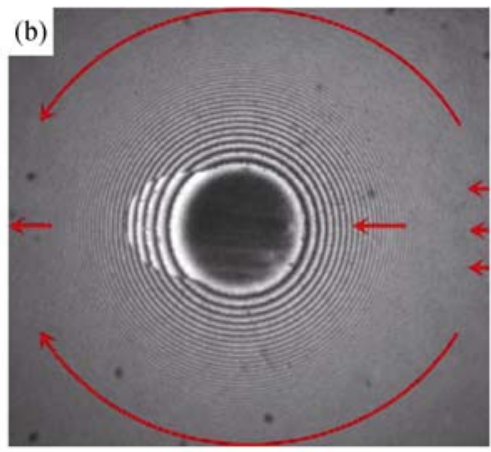

$15 \mathrm{~mm} / \mathrm{s}$

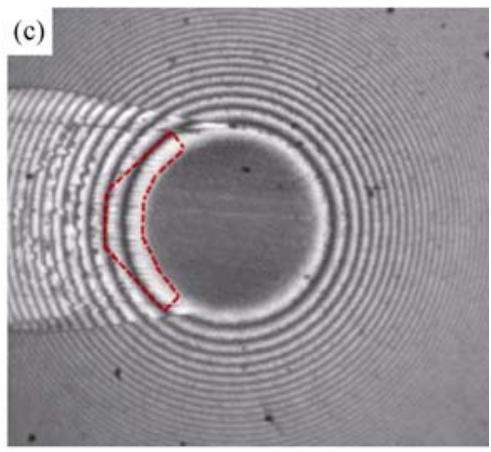

$100 \mathrm{~mm} / \mathrm{s}$

图 9 不同滑动速度下球盘点接触试验台接触区图像

Fig. 9 Pictures of the contact area on ball-on-disk tester at different sliding speeds

在典型的接触疲劳磨损以及黏着磨损产生的点蚀现 象,磨痕宽度为 $0.35 \mathrm{~mm}$ 。图 $8(\mathrm{~g})$ 和 $8(\mathrm{~h})$ 给出在机油 中添加微碳球后试样磨痕的表面形貌, 磨痕表面光 滑, 只能看见轻微的犁沟和微小的疲劳损伤, 磨痕 宽度也仅为 $0.27 \mathrm{~mm}$ 。

\section{4 润滑机理研究}

为了深入研究微碳球的润滑机理, 实验通过 球盘点接触试验台对相同载荷工况条件下含微碳 球的液体石蜡润滑接触区的润滑状态进行实时图 像跟踪记录, 结果如图 9 所示。其中, 图 9(a)表示 静止状态下的接触区图像, 在中心接触区周围可 肉眼观察到大量分散性良好的微碳球。图 9(b)表示 为对偶件之间产生相对运动时的接触区图像, 图 中标注的红线为微碳球的大致运动轨迹, 由于接 触区压力的存在, 大量微碳球在进入接触区之前 会绕过接触中心而从周围穿过, 只有少量微碳球 可以直接穿过润滑接触区。通过观察中间接触区颜 色的变化以及接触出口区存在的大量微碳球颗粒 可以发现, 在润滑过程中, 微碳球可以进入接触区 起到润滑作用。

随着相对速度的提高, 中心接触区颜色继续变 浅, 说明卷吸速度提高的同时也相应增加了中心膜 厚。在润滑出口区, 即图 9(c)中虚线位置, 可以明显 看到由于微碳球高速通过接触区而出现的类似于毛 刷形状的现象, 这证实了微碳球在润滑过程中的确 进入接触区起到特殊的减摩抗磨作用。

根据微碳球的自身性质以及相关摩擦实验结果, 我们认为微碳球在铝合金基底上的润滑行为主要是 由微碳球本身在接触区表面沉积造成的, 其作用机 理可用图 10 表示。在下试样表面粗䊁度的作用下, 微碳球颗粒被旋转的铝合金盘带入润滑接触区，同 时, 微碳球在接触区入口处不断地进行堆积和挤压,
使得微碳球更加容易进入摩擦区域参与润滑。进入 接触区的微碳球堆积在接触界面之间产生物理分离, 并将滑动摩擦一定程度的转化为滚动摩擦, 从而降 低摩擦, 减小磨损。

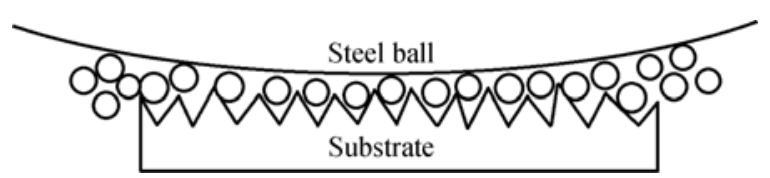

图 10 微碳球润滑机理示意图

Fig. 10 Schematic diagram of lubrication mechanism of carbon microspheres

\section{3 结论}

1) 通过葡萄糖水热法一步制备得到微碳球, 其粒径在 400 500 nm 之间, 呈现完美球形形状, 制 备过程简单, 绿色无污染, 易于工业化。

2) 微碳球具有特殊的摩擦学效应, 将其作为 液体石蜡和商业机油润滑添加剂在铝合金基底上表 现出优异的减摩耐磨性能。

3) 微碳球减摩耐磨作用机理在于滑动过程中 微碳球进入接触区域堆积在接触界面之间产生物理 分离，阻止摩擦副之间的直接接触，同时可以起到 纳米滚珠作用, 将滑动摩擦一定程度的转化为滚动 摩擦, 从而降低摩擦, 减小磨损。

\section{参考文献:}

[1] HEINZ A, HASZLER A, KEIDEL C, et al. Recent development in aluminium alloys for aerospace applications. Materials Science and Engineering: A, 2000, 280(1): 102-107.

[2] STARKE E A, STALEY J T. Application of modern aluminum alloys to aircraft. Progress in Aerospace Sciences, 1996, 32(2): 131-172. 
[3] MILLER W S, ZHUANG L, BOTTEMA J, et al. Recent development in aluminum alloys for the automotive industry. Materials Science and Engineering: A, 2000, 280(1): 37-49.

[4] LU P X, WEI D B, GUO C B, et al. Study on scanning micro-arc oxidation technology applied to 2024 aluminum alloy. Journal of Inorganic Materials, 2013, 28(4): 381-386.

[5] YE Z Y, LIU D X, LI C Y, et al. Effect of sealing treatments on the corrosion behavior of micro-arc oxidation coating on aluminum alloy in acid $\mathrm{NaCl}$ solution. Journal of Inorganic Materials, 2015, 30(6): 627-632.

[6] DWIVEDI D K. Adhesive wear behaviour of cast aluminium-silicon alloys: overview. Materials \& Design, 2010, 31(5): 2517-2531.

[7] GHAZALI M J, RAINFORTH W M, JONES H. The wear of wrought aluminium alloys under dry sliding conditions. Tribology International, 2007, 40(2): 160-169.

[8] DWIVEDI D K. Wear behaviour of cast hypereutectic aluminium silicon alloys. Materials \& design, 2006, 27(7): 610-616.

[9] MEHTA D S, MASOOD S H, SONG W Q. Investigation of wear properties of magnesium and aluminum alloys for automotive applications. Journal of Materials Processing Technology, 2004, 155: 1526-1531.

[10] RODRIGUEZ J, POZA P, GARRIDO M A, et al. Dry sliding wear behaviour of aluminium-lithium alloys reinforced with $\mathrm{SiC}$ particles. Wear, 2007, 262(3): 292-300.

[11] LIU W, XIA Y, XUE Q. Effect of P-N type extreme pressure and anti-wear additive on the friction and wear behaviour of aluminumsteel sliding pair. Tribology, 2000, 20(5): 331-335.

[12] LIU W M, HU Y H, HE Z M, et al. Friction and wear behaviour of an Al-Si alloy against steel lubricated with $\mathrm{N}$ - and O-containing organic compounds. Lubrication Science, 1998, 11(1): 37-49.

[13] HU Y, LIU W. Tribological properties of alcohols as lubricating additives for aluminum-on-steel contact. Wear, 1998, 218(2): 244-249.

[14] XIA Y, LIU W, XUE Q. Effect of several amide compounds as additives in liquid paraffin on friction and wear behavior of steel-steel and steel-aluminum alloy systems. Tribology, 2002, 22(1): 40-43.

[15] WAN Y, XUE Q, CAO L. Boundary lubrication of aluminum alloy with $\mathrm{Cl}$-containing antiwear and extreme pressure additives. Wear, 1997, 208(1): 57-60.

[16] WAN Y, XUE Q, LIU W. Tribological behavior of lubricating oil additives in lubricated aluminum-on-steel contact. Wear, 1996, 196(1): 87-91.

[17] WAN Y, XUE Q. An investigation of tribological properties of P-containing AW and EP additives in lubricated aluminum-on-steel contact. Acta Petrolei Sinica Petroleum Processing Section, 1996,
12: $108-114$.

[18] DAS S, BISWAS S K. Boundary lubricated tribology of an aluminium-silicon alloy sliding against steel. Tribology Letters, 2004, 17(3): 623-628.

[19] LIU X Q, ZHOU F, LIANG Y M, et al. Tribological performance of phosphonium based ionic liquids for an aluminum-on-steel system and opinions on lubrication mechanism. Wear, 2006, 261(10): 1174-1179.

[20] QU J, BLAU P J, DAI S, et al. Tribological characteristics of aluminum alloys sliding against steel lubricated by ammonium and imidazolium ionic liquids. Wear, 2009, 267(5): 1226-1231.

[21] MU Z, ZHOU F, ZHANG S, et al. Effect of the functional groups in ionic liquid molecules on the friction and wear behavior of aluminum alloy in lubricated aluminum-on-steel contact. Tribology International, 2005, 38(8): 725-731.

[22] JIMENEZ A E, BERMUDEZ M D, IGLESIAS P, et al. 1-N-alkyl-3-methylimidazolium ionic liquids as neat lubricants and lubricant additives in steel-aluminium contacts. Wear, 2006, 260(7): 766-782.

[23] HUANG H D, TU J P, GAN L P, et al. An investigation on tribological properties of graphite nanosheets as oil additive. Wear, 2006, 261(2): 140-144.

[24] NUNN N, MAHBOOBA Z, IVANOV M G, et al. Tribological properties of polyalphaolefin oil modified with nanocarbon additives. Diamond and Related Materials, 2015, 54: 97-102.

[25] STONE V, NOWACK B, BAUN A, et al. Nanomaterials for environmental studies: classification, reference material issues, and strategies for physico-chemical characterization. Science of the Total Environment, 2010, 408(7): 1745-1754.

[26] CAI P, FENG L. Synthesis of hollow carbon spheres by one convenient method. Materials Chemistry and Physics, 2008, 108(1): $1-3$.

[27] SHI L, GU Y, CHEN L, et al. Synthesis of carbon hollow spheres by a reaction of hexachlorobutadiene with sodium azide. Chemistry Letters, 2004, 33(5): 532-533.

[28] SUN X M, LI Y D. Colloidal carbon spheres and their core/shell structures with noble-metal nanoparticles. Angewandte Chemie (International Edition), 2004, 43(5): 597-601.

[29] ALAZEMI A A, ETACHERI V, DYSART A D, et al. Ultrasmooth submicrometer carbon spheres as lubricant additives for friction and wear reduction. ACS Applied Materials \& Interfaces, 2015, 7(9): 5514-5521.

[30] ST. DENNIS J E, JIN K, JOHN V T, et al. Carbon microspheres as ball bearings in aqueous-based lubrication. ACS Applied Materials \& Interfaces, 2011, 3(7): 2215-2218. 